\section{Hypopharyngeal foreign body masquerading as malignancy}

\author{
I. Rana, ${ }^{1}$ M. I. Syed, ${ }^{2}$ C. Adams ${ }^{3}$ and L. J. Clark ${ }^{4}$
}
IN BRIEF
- Pharyngeal foreign bodies can present without a positive history and can have a clinical presentation mimicking malignancy.
- A routine examination of a patient with dysphagia should include eliciting a specific history of wearing dentures and examination of teeth.
- In future designs for dental plates, bridges and crowns the use of a radio opaque material should be considered.

\begin{abstract}
Foreign body ingestion in dental and ENT practice is a commonly encountered emergency. In most cases, particularly in adults, there is a definite history of its ingestion, the nature of the foreign body is usually identifiable and the patient almost always presents immediately. We report an unusual case of an elderly patient with a six month history of progressive dysphagia referred to us by the physicians after investigations which were highly suggestive of a hypopharyngeal malignancy. Surprisingly when a biopsy was attempted, the hypopharyngeal mass turned out to be a dental plate. Dentists and otolaryngologists should be aware that pharyngeal foreign bodies can present without a positive history and can have a clinical presentation mimicking malignancy. A history of head injury, dementia, alcohol and drug abuse should be specifically excluded. A routine examination of a patient with dysphagia should include eliciting a specific history of wearing dentures and examination of teeth. In future designs for dental plates, bridges and crowns the use of a radio opaque material should be considered.
\end{abstract}

\section{CASE REPORT}

An 84-year-old male was referred to the ENT department by the physicians with a suspicion of a malignancy in the aerodigestive tract.

He was under their care for weight loss and recurrent chest infections. He had been having progressive dysphagia mainly for solids and throat discomfort for the last six months. He had a past history of alcohol abuse, heavy smoking and was under treatment for depression.

Routine blood results showed a normal full blood count but deranged liver function tests and nutritional deficiencies.

He underwent a barium swallow which showed some irregularity in the lateral wall of the hypopharynx (Fig. 1), and overall these findings were suggestive but not conclusive for malignancy. He

\footnotetext{
Senior House Officer in Otolaryngology, ${ }^{2 *}$ Specialist Registrar in Otolaryngology, ${ }^{4}$ Consultant in Otolaryngology, Southern General Hospital, Greater Glasgow \&t Clyde NHS trust, Glasgow, G51 3PZ; ${ }^{3}$ Consultant in Radiology, Royal Alexandra Hospital, Greater Glasgow \& Clyde NHS Trust, Paisley, PA2 9PN

*Correspondence to: Mr Mohammed lqbal Syed Email: iqbalms@hotmail.com
}

\section{Refereed Paper}

Accepted 15 July 2009

DOI: 10.1038/sj.bdj.2009.903

${ }^{\circledR}$ British Dental Journal 2009; 207: 361-362 was assessed by the speech and language therapists, who performed a video fluoroscopy, which showed that there was aspiration of thin fluids and a pharyngeal residue with no protective cough mechanism. A chest X-ray and abdominal ultrasound examination were unremarkable.

Examination of the palate was unremarkable, but the patient had poor dental hygiene and missing teeth $(21,22)$. On fibre optic laryngoscopy in the outpatient clinic, it was observed that there was pooling of saliva and a mass in the pyriform sinuses, highly suggestive of a malignancy. The rest of the examination was unremarkable.

Panendoscopy under general anaesthesia revealed a mass in both pyriform fossae and the post-cricoid region with exuberant granulations on the surface. Surprisingly when a biopsy was attempted the mass appeared to be hard and partially mobile. On further manipulation a foreign body was found to be snugly fitting in both pyriform fossae and the post cricoid area.

This was removed under direct vision and was found to be a large dental plate which was $4.5 \times 4.0 \mathrm{~cm}$ in dimension (Fig. 2). The rest of the examination was normal but for chronic inflammation and an indentation in the lateral wall of the right pyriform sinus, caused by the dental

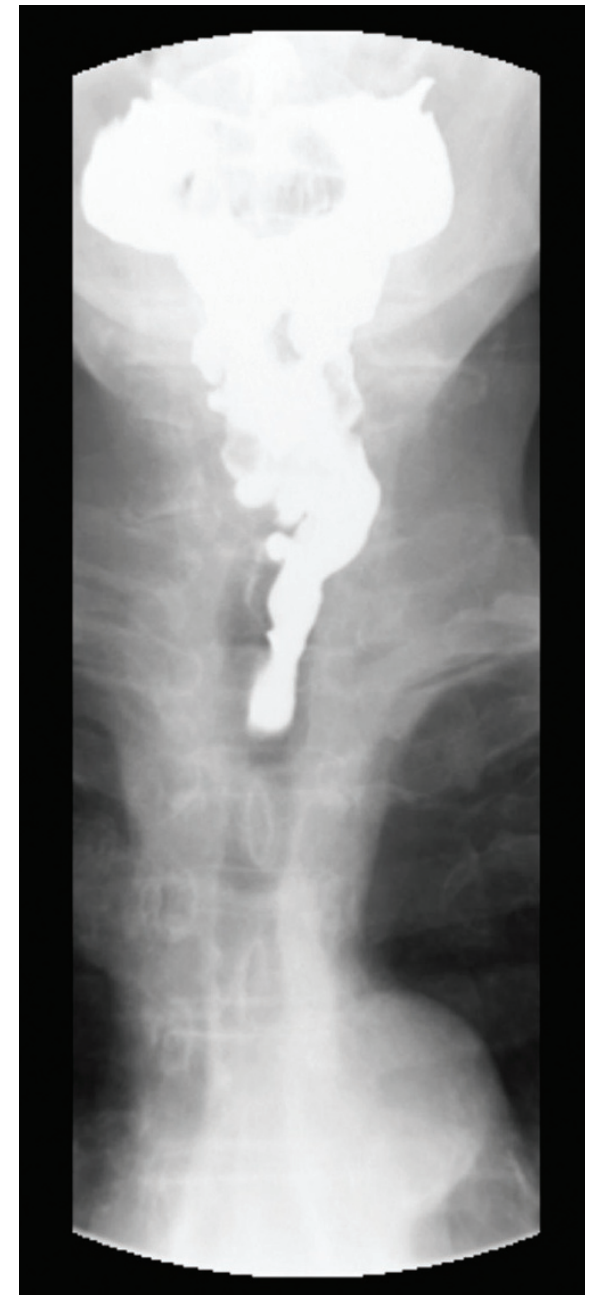

Fig. 1 Barium swallow showing irregularity in the lateral wall of hypopharynx 
plate. The patient was fed via a nasogastric tube for the next two days, which was inserted during the procedure as a precautionary measure.

The patient recovered well and was finally discharged home, after an appointment with a dentist was arranged. At his local dentist the patient was fitted with a case partial denture with clasps for better retention.

\section{DISCUSSION}

The incidence of impacted foreign body in the form of a dental prosthesis is $0.4 \%{ }^{1}$ to $0.7 \%{ }^{2}$ and in most cases they are partial dentures as opposed to full dentures or crowns.

In the fully conscious adult accidental dislodgement and ingestion of a dental prosthesis can occur while eating or drinking, giving rise to acute onset of odynophagia, choking or retrosternal pain and these patients present early, and give a definitive history.

In patients with history of altered consciousness such as head injury, stroke, seizures, alcohol abuse or dementia, there may not be a history available, and the diagnosis may easily be missed. Occasionally these patients may present with complications such as an oesophageal perforation, neck/lung abscesses, mediastinitis or oesophago-aortic fistula, which in turn leads to the diagnosis.

Our patient did have a history of alcohol abuse, but surprisingly did not have any acute symptoms, and presented with progressive dysphagia and weight loss, which were more suggestive of a malignancy rather than foreign body ingestion. On specifically questioning the patient post-operatively he did remember that his dentures had been missing for almost a year.

In our patient the dental plate was lodged in the hypopharynx which is a relatively uncommon site for impaction of dentures. In a case series of 21 patients with ingested dental prosthesis, Abdullah et al. ${ }^{3}$ found the hypopharynx was the site in three cases, as opposed to cervical oesophagus

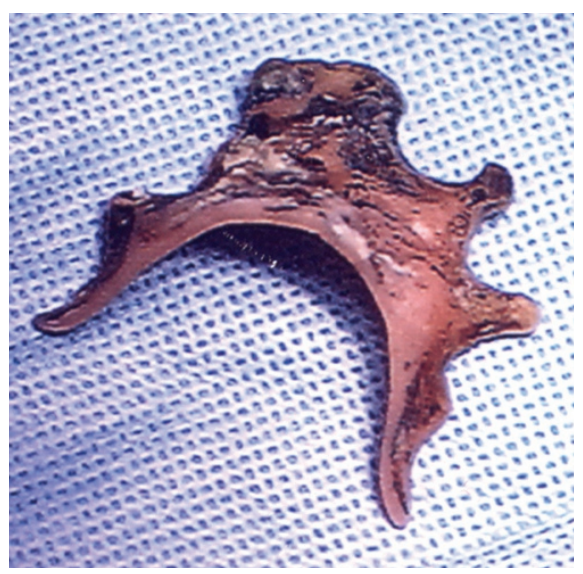

Fig. 2 Dental plate $(4.5 \times 4.0 \mathrm{~cm})$ removed from the hypopharynx

(9/21), mid and lower oesophagus (6/21), rest of gastrointestinal tract (3/21).

Poly (methylmethacrylate), the plastic from which most dentures are made, is radiolucent. Porcelain teeth produce light shadows on a plain radiograph but it is the metal pins attaching the teeth to the denture base that make them readily discernible. However, with the improvement in appearance and wear resistance of the better quality plastic and composite artificial teeth, porcelain teeth are seldom used on dentures in the UK. ${ }^{4}$ Despite the many calls for use of radio-opaque denture base materials ${ }^{5}$ no such product seems available in the UK. Tsao ${ }^{6}$ and Davy ${ }^{7}$ investigated the use of materials in dentures to make them radio-opaque, without adversely compromising on the strength of the material, but these were never marketed because of concerns that the halide in the material might have cancer-inducing potential.

Radiology and endoscopy are the key investigations in patients with suspected foreign body ingestion or dysphagia caused by suspected malignancy.

Although a plain soft tissue X-ray of the neck may not identify a swallowed denture, it can exclude pneumomediastinum or gas within the soft tissues. Barium swallow is usually one of the early investigation modalities done in patients with swallowing problems. Many authors have quoted a high sensitivity and specificity of barium study in the detection of ingested foreign bodies $^{8}$ whereas others ${ }^{4}$ have found it to be unhelpful, making subsequent endoscopy more difficult. In our case barium swallow was inconclusive. It has been observed that higher contrast and spatial resolution of CT scan enables better detection and localisation of foreign bodies along with soft tissue changes caused by the trauma from the foreign body. ${ }^{9}$

Our patient was scheduled for a CT scan after the panendoscopy, but this was subsequently cancelled as the cause for dysphagia was immediately found during the procedure.

\section{CONCLUSION}

Accidental ingestion of dentures is not unusual and such patients present acutely, however, there are patients that may have a delayed atypical presentation. Dentists and otolaryngologists should be aware that certain individuals may not necessarily give a history or report the disappearance of a denture.

A routine examination of a patient with dysphagia should include eliciting a specific history of wearing dentures and examination of teeth.

In future designs for dental plates, bridges and crowns the use of a radio opaque material should be considered.

1. Marais J, Mitchell R, Wightman A J A. The value of radiographic assessment of oropharyngeal foreign bodies. J Laryngol Otol 1995; 109: 452-454.

2. Nandi P, Ong G B. Foreign body in the oesophagus: review of 2,394 cases. Br J Surg 1978; 65: 5-9.

3. Abdullah B J J, Teong L K, Mahadevan J, Jalaludin A. Dental prosthesis ingested and impacted in the esophagus and orolaryngopharynx. J Otolaryngol 1998; 27: 190-194.

4. Hashmi S, Walter J, Smith W, Latis S. Swallowed partial dentures. J R Soc Med 2004; 97: 72-75.

5. Chandler $H \mathrm{H}$, Bowen R L, Paffenbarger G C. Development of a radio-opaque denture base material. J Biomed Mater Res 1971; 5: 253-265.

6. Tsao D H, Guilford H J, Kazanoglu A, Bell D H. Clinical evaluation of a radio-opaque denture base resin. J Prosthet Dent 1984; 51: 456-458.

7. Davy K W, Causton B E. Radio-opaque denture base: a new acrylic polymer. J Dent 1982; 10: 254-264.

8. Loh LE, Chee S G. Barium swallow: Its role in the management of an oesophageal F B. Singapore Med J 1988; 29: 438-440.

9. Braverman I, Gomori J M, Polv O, Saah D. The role of CT imaging in the evaluation of cervical esophageal foreign bodies. J Otolaryngol 1993; 22: 311-314. 